simply using regular medical skills.

The author suggests that international colleagues support each other in large disasters and develop academic exchanges so as to improve emergency care organization and skills.

Key Words: deaths; disabilities; earthquake; injuries; Rescue Emergency Medicine

\section{A Nuclear Disaster Handling Manual}

Y. Haragucbi; H. Nishi; Y. Tomoyasu; T. Arai

Clinical Research Institute, National Hospital Tokyo

Disaster Medical Center, Tachikawa City, Tokyo, Japan

This March 1997, a large scale of accident in a nuclear waste plant occurred in Tokaimura, which is located at a distance of approximately $150 \mathrm{~km}$ from the center of Tokyo. More than 30 people who were working in the plant were contaminated. This accident reminds us of the potential for the occurrence of mass casualties by radioactive substances.

In the event of a disaster, the National Hospital Tokyo Disaster Medical Center will take charge of disaster-related medical care. In this Institute, we have been developing a disaster manual that focuses on the handling of casualties subjected to nuclear contamination. The development of this manual is discussed.

At the present, many problems remain when treating casualties contaminated with radioactive substances. Among them was the difficulty of determining the existence of the contamination with accuracy, effective methods for decontamination of the victims, the use of drugs that will promote excretion of toxic substances, transportation of patients, special rooms where contaminated patients are admitted, the storage and disposal of the polluted materials and water, and legal problems

Key Words: contamination; manual decontamination; nuclear disaster; radioactive substances

\section{Analysis of the Kinds of Diseases Encountered in the Shanghai Prehospital Emergency Care Service: 1991-1995 \\ $X u$ Huiliang \\ National Training Center for Emergency Care Personnel, Shanghai, Peoples Republic of China,}

The author analyzed 467,674 cases of prehospital emergency care provided by the Shanghai Medical First-Aid Center during 1991-1995. The aim of this analysis was to determine the disease spectrum of prehospital emergency care provided in Shanghai, and to identify ways by which to improve the level of prehospital emergency care.

Results: There were 268,300 males cases $(57.3 \%)$ and 199,374 female cases $(42.6 \%)$. The ratio of male to female was $1: 345.49 .2 \%$ of the patients were $\geq 60$ years of age; $20 \%$ of the cases were critically diseased or injured. The five leading causes that demanded prehospital emergency care in rank order were: 1) traumatic injuries; 2) cerebrovascular diseases; 3) cardiac diseases; 4) acute abdominal pain; and 5) respiratory diseases. The five leading causes of prehospital deaths were: 1) cardiac diseases; 2) suicides and intoxication; 3) respiratory emergencies; 4) cerebrovascular diseases; and 5) neoplasms. The results also showed the characteristics of monthly distribution of several disorders.

Conclusion: Cardiac diseases, cerebrovascular diseases, respiratory tract diseases, injuries, and intoxication should be the focal points for prehospital emergency care instruction, and ambulance equipment and drug supplies should fit the demands of these diseases and fit the seasonal changes in disease spectrum.

Key Words: causes of death; disease distribution; prehospital emergency care; Shanghai

\section{The Disastrous Epidemic 0-157}

(Escherichia Coli) Food Poisoning in Osaka

Arito Kaji; Tosbinori Miyaicbi; Hirosbi Rinka;

Yoshio Matsuo; Masanori Kan; Tatsubiro Shigemoto;

Takahisa Yosbimura; Kazuma Tsukioka; Takashi Ukai

Emergency and Critical Care Medical Center, Osaka

City General Hospital, Osaka, Japan

In July 1996, more than 6,000 children suffered from acute food poising caused by Enterohemorrhagic Escherichia Coli-0-157 after eating school lunches in Sakai City of the Osaka Prefecture. Sakai City has a population of approximately 900,000 and neighbors Osaka City (about $20 \mathrm{~km}$ to the south).

Severe diarrhea, vomiting, and bloody stools led some children to consult local physicians on 11 July, but the number of victims increased abruptly on 13 July (Saturday), and overwhelmed the capacity of pediatric medical services in Sakai. The number of children who needed admission was 32 on $13 \mathrm{July}$, and increased to 493 on the next day. Hospitals in Sakai were so crowded with the ill children that some of the children had to wait 6 hours for consultation. On the other hand, hospitals in Osaka City (neighboring city to Sakai) were not crowded, and the out-patient departments and wards were quiet as in a normal weekend.

The Osaka Prefecture Emergency Medical Information Center (OPEMIC) looked for vacant beds in all of the Osaka Prefecture to Sakai Fire Department and Health Bureau of Sakai City Government from the evening of 13 July; 152 patients were transferred to 44 hospitals in other cities. Osaka City General Hospital (OCGH) received 15 moderately ill patients in the first two days. As all the pediatric beds of OCGH were occupied on the afternoon of 13 July, six additional beds were prepared in the emergency center. Eight more patients were transferred to OCGH by the request of OPEMIC. In total, 23 children were admitted and six of them were seriously ill due to dehydration and/or acute renal failure (hemolytic uremic syndrome).

All of the patients recovered within one month without any sequellae. However, three children treated in other hospitals died from Hemolytic Uremic Syndrome and encephalopathy.

This disastrous epidemic was inadequately recognized. The congestion and the confusion of the hospitals in Sakai in the first few days could have been avoided. 